\title{
Direct effects of CPT-11 and SN38 on ovarian granulosa cells
}

\author{
TOMOKO UTSUNOMIYA $^{1 *}$, TETSUJI TANAKA ${ }^{1 *}$, HIROTOSHI UTSUNOMIYA $^{2}$ and NAOHIKO UMESAKI ${ }^{1}$ \\ Departments of ${ }^{1}$ Obstetrics and Gynecology, and ${ }^{2}$ Experimental Pathology, Wakayama \\ Medical University, 811-1 Kimi-idera, Wakayama 641-0012, Japan
}

Received October 30, 2008; Accepted December 16, 2008

DOI: 10.3892/mmr_00000082

\begin{abstract}
This study aimed to clarify the mechanism by which apoptosis and Fas ligand (FasL) expression are induced in the ovarian granulosa cells of mice injected with irinotecan $\mathrm{HCl}$ (CPT-11). To this end, the direct effects of CPT-11 and its active metabolite, $\mathrm{SN} 38$, on granulosa cells were investigated. Normal ovarian tissue fragments obtained from 8-week-old female $\mathrm{MCH}$ mice were cultured in vitro with $\mathrm{CPT}-11$ or SN38 and paraffin-embedded. After sectioning, the ovarian fragments were analyzed by TUNEL staining to detect apoptotic cells and by immunohistochemistry with an antiFasL antibody to detect FasL expression. The results revealed no increase in TUNEL-positive granulosa cells in the ovarian tissue fragments cultured with CPT-11 or SN38. Furthermore, CPT-11 and SN38 did not induce FasL expression in the ovarian fragments. In conclusion, apoptosis and FasL expression induced in the ovarian granulosa cells of mice injected with CPT-11 is not caused by direct stimulation with CPT-11 or SN38. Therefore, systemic CPT-11 administration appears to induce apoptosis and FasL expression in granulosa cells via currently unknown endogenous FasL-inducing factors or by active metabolites of CPT-11 other than SN38.
\end{abstract}

\section{Introduction}

The anticancer drug irinotecan $\mathrm{HCl}$ (CPT-11) is converted into its main active metabolite, SN38, by carboxyl esterase in the body (1). SN38 is a powerful inhibitor of topoisomerase I and exhibits strong antitumor effects by antagonizing DNA synthesis (1). CPT-11 has been used clinically in various types of cancer chemotherapy, including that against uterine (2-4), ovarian (5-6), lung (7), colorectal (8-9) and gastric cancer (10),

Correspondence to: Dr Tetsuji Tanaka, Department of Obstetrics and Gynecology, Wakayama Medical University, 811-1 Kimi-idera, Wakayama 641-0012, Japan

E-mail: obgywmu@wakayama-med.ac.jp

${ }^{*}$ Contributed equally

Key words: apoptosis, granulosa cell, irinotecan, SN38, Fas ligand, ovarian failure, topoisomerase I inhibitor as well as malignant lymphoma (11). High response rates to these therapies have been noted. Recently, we reported that pre- and perimenopausal cancer patients aged approximately 50 years and treated with CPT-11 combination chemotherapy frequently complained of menopausal malaise-like symptoms (MMLS), such as hot flashes, episodic sweating, peripheral chill, irritability and insomnia (12-13). In these patients, MMLS were completely relieved by estrogen therapy, indicating that they were induced by estrogen deficiency. In animal experiments using CPT-11-injected mice, Fas ligand (FasL) expression was specifically induced in the granulosa cells of ovarian large follicles and reacted with Fas antigens constitutively expressed on the granulosa cells, thereby inducing granulosa cell apoptosis (14). This is considered to represent one of the main mechanisms of CPT-11-induced ovarian failure in cancer patients. However, the mechanisms underlying the induction of apoptotis and FasL expression in the granulosa cells of CPT-11-injected mice remain unknown.

Regarding the development of novel preventive therapies for anticancer drug-induced ovarian failure, it is important to identify the factors that directly induce apoptosis and FasL expression in the granulosa cells of CPT-11-injected mice. Although an intraperitoneal injection of CPT-11 dosedependently induces ovarian follicular apoptosis in mice (14), CPT-11 itself has very weak cytotoxic effects against cancer cells (1). Its metabolite, SN38, is considered to be the strongest antitumor molecule derived from CPT-11. Therefore, in the present study, we investigated the key factors involved in the direct induction of apoptosis and FasL expression in granulosa cells using organ culture experiments.

\section{Materials and methods}

Oophorectomy of mice after intraperitoneal injection of CPT-11. Animal experiments were carried out using 8-weekold female MCH mice (Nihon Clea Co. Ltd., Osaka, Japan). Each mouse received one intraperitoneal injection of CPT-11 (100 $\mu \mathrm{g}$; Yakult Co., Ltd., Tokyo, Japan) in saline $48 \mathrm{~h}$ after an intraperitoneal injection of pregnant mare serum gonadotropin (5 units; Teikoku Zouki Co., Tokyo, Japan) to create similar ovarian conditions among the mice. Control mice received an intraperitoneal injection of saline rather than CPT-11. Under ether anesthesia, the abdomen was opened and the ovaries were dissected $24 \mathrm{~h}$ after CPT-11 administration. The isolated ovaries were fixed with $0.2 \%$ paraformaldehyde on ice overnight and embedded in paraffin. 
in vitro organ culture

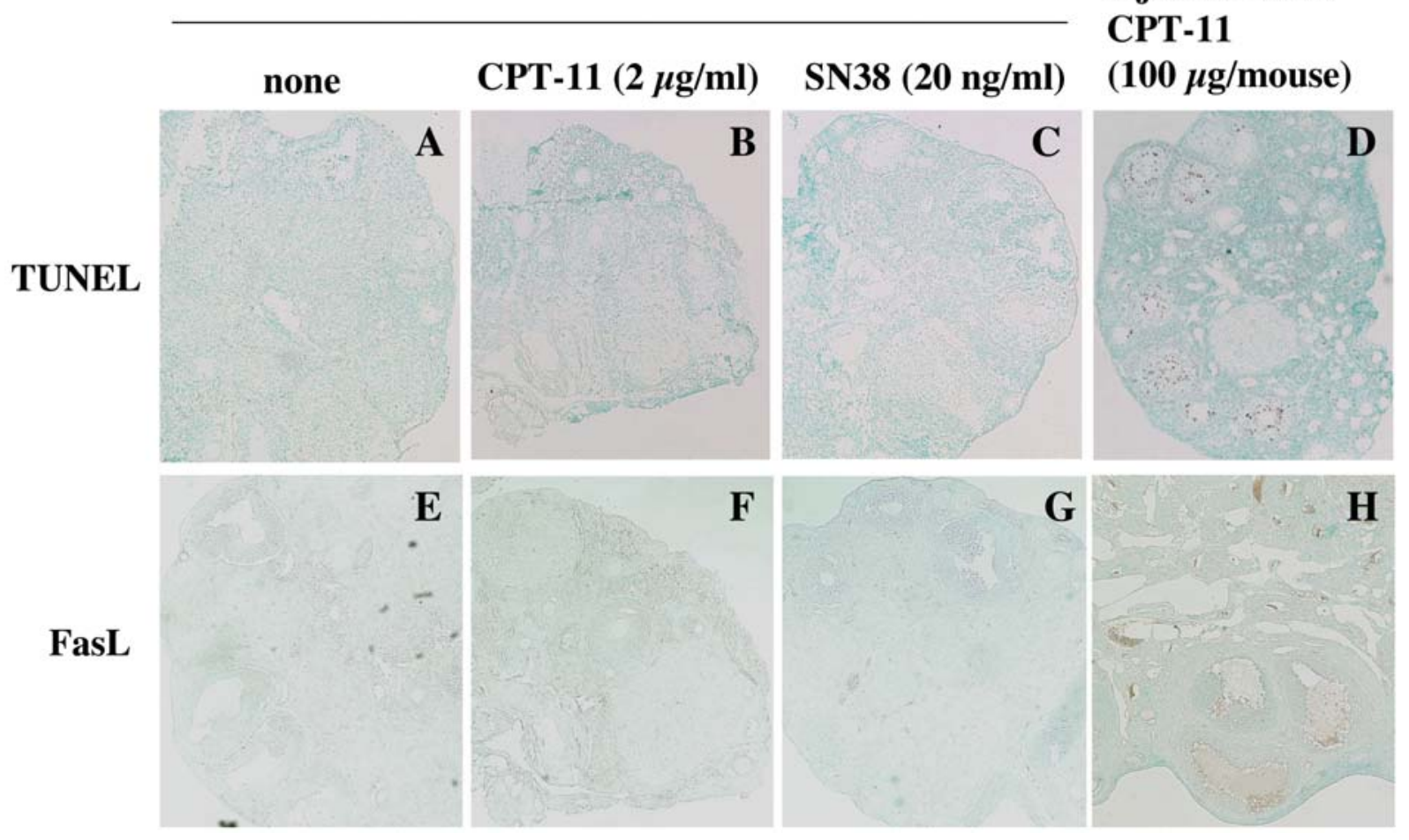

Figure 1. TUNEL staining and immunohistochemical analysis of ovarian tissues after in vitro culture with CPT-11 or SN38. (A-D) TUNEL staining. (E-H) Immunohistochemistry for FasL expression. (A-C) and (E-G) in vitro-cultured ovarian fragments. (D and H) Ovaries excised from mice injected intraperitoneally with CPT-11. Almost no TUNEL-positive follicles were detected in the (B) CPT-11-treated or (C) SN38-treated ovarian tissues. No FasL-positive follicles are present in the (F) CPT-11-treated or (G) SN38-treated ovarian tissues. The ovaries from mice injected with CPT-11 contain (D) TUNEL-positive and (H) FasLpositive follicles.

In vitro organ culture of normal ovarian tissue fragments. Normal ovaries were removed from 8-week-old female $\mathrm{MCH}$ mice $48 \mathrm{~h}$ after an intraperitoneal injection of pregnant mare serum gonadotropin (5 units), cut into thin longitudinal fragments and cultured in vitro in 384-well culture plates (Genetix Ltd., New Milton, Hampshire, UK) in OPTI-MEM (Gibco-BRL, Gaithersburg, MD, USA) supplemented with $5 \%$ fetal calf serum (Equitech Bio Inc., Ingram, TX, USA), $100 \mathrm{U} / \mathrm{ml}$ penicillin (Gibco-BRL) and $100 \mu \mathrm{g} / \mathrm{ml}$ streptomycin (Gibco-BRL). CPT-11 or SN38 was added to the ovarian fragments and incubated for $5 \mathrm{~h}$ at $37^{\circ} \mathrm{C}$ in a $5 \% \mathrm{CO}_{2} / 5 \%$ $\mathrm{O}_{2} / 90 \% \mathrm{~N}_{2}$ atmosphere. The final concentrations of CPT-11 were $0,0.2$ and $2 \mu \mathrm{g} / \mathrm{ml}$, while those of SN38 were 0,2 and $20 \mathrm{ng} / \mathrm{ml}$. For each experiment, six ovarian fragments from six ovaries were used. After the culture, these fragments were fixed with $0.2 \%$ paraformaldehyde in phosphate-buffered saline (PBS) overnight on ice and embedded in paraffin. Following sectioning, the paraffin-embedded tissues were analyzed by TdT-mediated dUTP-biotin nick end-labeling (TUNEL) staining to detect apoptotic cells, or were subjected to immunohistochemistry for FasL expression as described below. Ovarian follicles within the tissue sections obtained from the six ovaries were counted.

TUNEL staining of ovarian sections. Paraffin-embedded ovarian tissue sections were deparaffinized, treated with proteinase K $(20 \mu \mathrm{g} / \mathrm{ml}$; Roche Diagnostic, Mannheim, Germany) for $10 \mathrm{~min}$ at room temperature and washed four times with distilled water for 5 min each at room temperature. Endogenous peroxidase activity in the sections was then blocked by incubation with $3 \%$ hydrogen peroxide in PBS for $5 \mathrm{~min}$ at room temperature. Finally, the sections were subjected to TUNEL staining using an ApopTag peroxidase in situ apoptosis detection kit (Serologicals Corporation, Billerica, MA, USA). TUNEL-positive follicles were defined as follicles containing at least 10 TUNEL-positive cells within an ovarian section. Experiments were performed twice to verify the results. The number of apoptotic and total follicles within the tissue sections obtained from six ovaries was counted, and the ratio of apoptotic to total follicles was calculated.

Immunohistochemistry for FasL expression. Paraffinembedded tissue sections were deparaffinized and treated with $0.3 \%$ hydrogen peroxide in methanol to block endogenous peroxidase activity. Following antigen retrieval by microwave treatment at $100^{\circ} \mathrm{C}$ for $15 \mathrm{~min}$ in citrate buffer $(1.8 \mathrm{mM}$ $\mathrm{C}_{6} \mathrm{H}_{8} \mathrm{O}_{7}, 8.2 \mathrm{mM} \mathrm{Na}_{3} \mathrm{C}_{6} \mathrm{H}_{5} \mathrm{O}_{7}, \mathrm{pH} 6.0$ ), the sections were incubated with anti-mouse FasL polyclonal rabbit $\operatorname{IgG}(1: 1$; Spring Bioscience, Fremont, CA, USA) at $4^{\circ} \mathrm{C}$ overnight, washed four times with PBS for 5 min each at room temperature, and incubated with a peroxidase-conjugated anti-rabbit secondary antibody (Simple Stain Max PO kit; Nichirei Co., Tokyo, Japan) for $30 \mathrm{~min}$ at room temperature. Finally, the sections were washed four times with PBS for $5 \mathrm{~min}$ each at room temperature, incubated with diaminobenzidine (Dako, Carpinteria, CA, USA) solution comprising of $30 \mathrm{mg}$ 


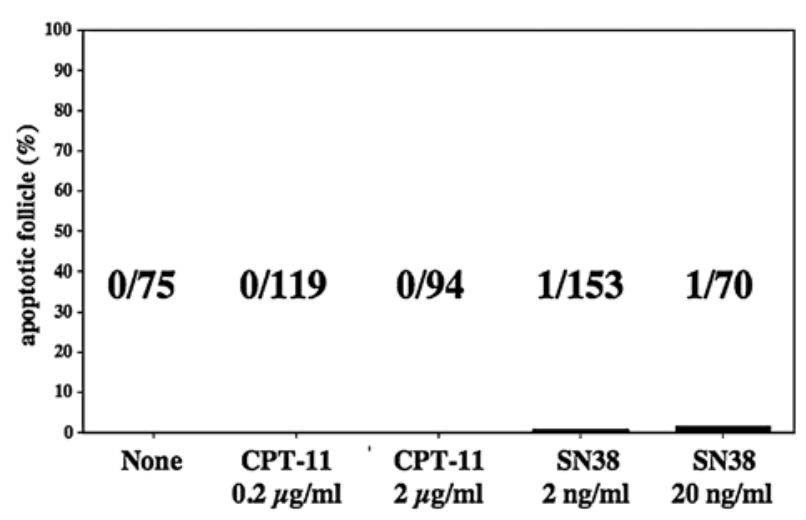

Figure 2. TUNEL-positive follicle ratios in ovarian tissues after in vitro culture with CPT-11 or SN38. Six ovarian fragments were cultured in vitro with CPT-11 or SN38 for $5 \mathrm{~h}$. The number of total and TUNEL-positive follicles was counted, and the ratio of TUNEL-positive to total follicles calculated. In the six ovaries incubated with $2 \mathrm{ng} / \mathrm{ml} \mathrm{SN} 38$ for $5 \mathrm{~h}$, only one TUNELpositive follicle was detected among 153 ovarian follicles examined $(<1 \%)$.

diaminobenzidine in $150 \mathrm{ml}$ of $0.05 \mathrm{M}$ Tris- $\mathrm{HCl}(\mathrm{pH} 7.6)$ containing $4 \%$ hydrogen peroxide and counterstained with methyl green (Nakalai Tesque Co. Ltd., Kyoto, Japan) at room temperature. FasL-positive follicles were defined as follicles containing at least 10 FasL-positive cells within an ovarian section. All experiments were performed twice to verify the results. The number of FasL-positive and total follicles within the tissue sections obtained from six ovaries was counted, and the ratio of apoptotic to total follicles was calculated.

\section{Results}

TUNEL-positive follicles in ovarian tissue fragments incubated with CPT-11 or SN38. Ovarian tissue fragments incubated with CPT-11 or SN38 were subjected to TUNEL staining to detect apoptotic cells. As shown in Fig. 1A-C, almost no TUNEL-positive follicles (containing $\geq 10$ TUNEL-positive granulosa cells) were observed in either CPT-11- or SN38treated ovarian fragments, whereas large follicles in the ovaries from the CPT-11-injected mice were TUNEL-positive (Fig. 1D). The number of TUNEL-positive follicles and total follicles in tissue sections obtained from six ovaries was counted, and the ratio of apoptotic follicles to total follicles was compared. As shown in Fig. 2, no TUNEL-positive follicles were detected in six non-treated ovaries and six CPT-11treated ovaries after $5 \mathrm{~h}$ of culture. Only one TUNEL-positive follicle was detected among 70 follicles examined in six ovaries incubated with $20 \mathrm{ng} / \mathrm{ml} \mathrm{SN38}$. Similarly, only one TUNEL-positive follicle was observed among 153 follicles examined in six ovaries incubated with $2 \mathrm{ng} / \mathrm{ml} \mathrm{SN} 38$.

FasL-positive follicles in ovarian tissue fragments incubated with CPT-11 or SN38. Ovarian tissue fragments incubated with CPT-11 or SN38 were evaluated for FasL expression by immunohistochemistry with an anti-FasL antibody. As shown in Fig. 1E-G, no FasL-positive follicles (containing $\geq 10$ FasLpositive granulosa cells) were observed in either CPT-11- or SN38-treated ovarian fragments, whereas large and medium follicles in the ovaries from the intraperitoneally CPT-11injected mice were FasL-positive (Fig. 1H).

\section{Discussion}

Ovarian failure caused by anticancer chemotherapy is usually irreversible, and cancer patients with post-chemotherapy ovarian failure often complain of estrogen deficiency symptoms or incurable sterility. However, the molecular mechanisms of anticancer drug-induced ovarian failure have yet to be clarified, and preventive therapies remain to be developed. A recent clinical endocrinological analysis revealed that CPT-11-combined chemotherapy often induces secondary amenorrhea and menopause in cancer patients (13). We previously demonstrated that one of the main mechanisms of CPT-11-induced ovarian failure is CPT-11-induced granulosa cell apoptosis in large ovarian follicles (14). Moreover, we showed that CPT-11-induced FasL expression in granulosa cells represents the main cause of CPT-11-induced granulosa cell apoptosis (14). These findings suggest that some ovarian failure induced by anticancer drugs may be prevented by inhibiting the induction of FasL expression in granulosa cells.

To develop preventive therapies for drug-induced ovarian failure, the key molecules inducing ovarian follicular injuries need to be identified. Therefore, in the present study, we examined whether CPT-11 or its metabolite SN38 directly induced granulosa cell apoptosis and/or FasL expression in granulosa cells. However, neither CPT-11 nor SN38 induced apoptosis or FasL expression in granulosa cells in our organ culture experiments. The incubation of ovarian tissues with CPT-11 and SN38 was carried out for $5 \mathrm{~h}$ in these organ cultures because overnight organ cultures produced spontaneous follicular destruction, and drug-induced ovarian apoptosis could not be evaluated. In rats intravenously injected with ${ }^{14} \mathrm{C}-\mathrm{CPT}-11$ (10 mg/kg body weight), the mean concentration of ${ }^{14} \mathrm{C}$-CPT-11 derivatives in the ovaries $4 \mathrm{~h}$ after the injection was reported to be $1.9 \mu \mathrm{g} / \mathrm{g}$ tissue weight $(15-16)$. The ${ }^{14} \mathrm{C}$ CPT-11 derivatives did not accumulate in either the testes or ovaries as compared with ${ }^{14} \mathrm{C}$ concentrations in the extragonadal organs. Intravenously injected CPT-11 is metabolized to its most active metabolite, SN38, within $30 \mathrm{~min}$ (17). Since most clinically administered CPT-11 disappears from the bloodstream within a few hours (17-18), the intraperitoneal injection of CPT-11 at $100 \mu \mathrm{g} /$ mouse ( 18-20 g body weight) represents a weaker stimulation than $5 \mathrm{~h}$ of direct exposure of ovarian fragments to high doses of CPT-11 or SN38. It is therefore highly unlikely that CPT-11 and SN38 are direct inducers of apoptosis and FasL expression in granulosa cells.

In a mouse in vivo study (14), CPT-11 increased apoptotic follicles in a dose-dependent manner. As neither CPT-11 nor SN38 is a direct inducer of apoptosis and FasL expression in granulosa cells, it is possible that CPT-11 stimulates tertiary factors in dose-dependent manners to induce apoptosis and FasL expression. Two possible hypotheses regarding such tertiary factors are proposed. First, unknown CPT-11-derived active metabolites other than SN38 (19) may directly induce apoptosis and/or FasL expression in granulosa cells. Second, unknown endogenous molecules stimulated by CPT-11 and/or SN38 may directly induce apoptosis and/or FasL expression in granulosa cells. However, the latter hypothesis is unlikely because no physiological ovary-specific apoptotic inducers have been identified to date. The identification of such factors is currently under investigation. 


\section{Acknowledgements}

This study was supported in part by a Grant-in-Aid for Scientific Research from the Ministry of Education, Science, Sports and Culture of Japan.

\section{References}

1. Kawato Y, Aonuma M, Hirota Y, Kuga $\mathrm{H}$ and Sato K: Intracellular roles of SN-38, a metabolite of the camptothecin derivative CPT-11, in the antitumor effect of CPT-11. Cancer Res 51: 4187-4192, 1991.

2. Sugiyama T, Yakushiji M, Noda K, et al: Phase II study of irinotecan and cisplatin as first-line chemotherapy in advanced or recurrent cervical cancer. Oncology 58: 31-37, 2000.

3. Umesaki N, Fujii T, Nishimura R, et al: Phase II study of irinotecan combined with mitomycin-C for advanced or recurrent squamous cell carcinoma of the uterine cervix: the JGOG study. Gynecol Oncol 95: 127-132, 2004.

4. Tanaka T, Kokawa K and Umesaki N: Preoperative chemotherapy with irinotecan and mitomycin for FIGO stage IIIb cervical squamous cell carcinoma: a pilot study. Eur J Gynaec Oncol 26: 605-607, 2005.

5. Tanaka T, Umesaki N and Ogita S: Camptothecin and mitomycin combination chemotherapy on ovarian clear cell carcinoma with multiple systemic metastases. Eur J Gynaec Oncol 11: 377-379, 2000 .

6. Nishino K, Aoki Y, Amikura T, et al: Irinotecan hydrochloride (CPT-11) and mitomycin $\mathrm{C}$ as the first line chemotherapy for ovarian clear cell adenocarcinoma. Gynecol Oncol 97: 893-897, 2005.

7. Noda K, Nishiwaki Y, Kawahara M, et al: Irinotecan plus cisplatin compared with etoposide plus cisplatin for extensive small-cell lung cancer. N Eng J Med 346: 85-91, 2002.

8. Saltz LB, Cox JV, Blanke C, et al: Irinotecan plus fluorouracil and leucovorin for metastatic colorectal cancer. N Eng J Med 343: 905-914, 2000
9. Douillard JY, Cunningham D, Roth AD, et al: Irinotecan combined with fluorouracil compared with fluorouracil alone as first-line treatment for metastatic colorectal cancer: a multicentre randomized trial. Lancet 355: 1041-1047, 2000.

10. Yamao T, Shirao K, Matsumura Y, et al: Phase I-II study of irinotecan combined with mitomycin-C in patients with advanced gastric cancer. Annal Oncol 12: 1729-1735, 2001.

11. Tobinai $\mathrm{K}$ and Hotta T: Clinical trials for malignant lymphoma in Japan. Jpn J Clin Oncol 34: 369-378, 2004.

12. Tanaka T, Umesaki N and Ogita S: Effects of shakuyaku-kanzoto on menopausal symptoms in an ovarian cancer patient treated with irinotecan HCl. J Trad Med 15: 456-457, 1998.

13. Tanaka $\mathrm{T}$, Utsunomiya $\mathrm{T}$, Utsunomiya $\mathrm{H}$ and Umesaki $\mathrm{N}$ : Irinotecan $\mathrm{HCl}$, an anticancer topoisomerase I inhibitor, frequently induces ovarian failure in premenopausal and perimenopausal women. Oncol Rep 19: 1123-1133, 2008.

14. Utsunomiya $T$, Tanaka $T$, Utsunomiya $H$ and Umesaki N: A novel molecular mechanism for anticancer drug-induced ovarian failure: Irinotecan $\mathrm{HCl}$, an anticancer topoisomerase I inhibitor, induces specific FasL expression in granulosa cells of large ovarian follicles to enhance follicular apoptosis. Int J Oncol 32: 991-1000, 2008.

15. Hakusui H, Suzuki W, Atsumi R, et al: Studies on the metabolic fate of CPT-11 (1): pharmacokinetics in female rats following a single intravenous dose (1). Xenobio Metab Dispos 6: 73-95, 1991.

16. Atsumi R, Suzuki W, Hakusui H, et al: Studies on the metabolic fate of CPT-11 (4): pharmacokinetics in female rats following a single intravenous dose. Xenobio Metab Dispos 6: 137-149, 1991.

17. Kawato Y, Aonuma M, Matsumoto K and Sato K: Production of SN-38, a main metabolite of the camptothecin derivative CPT-11, and its species and tissue specificities. Xenobio Metab Dispos 6: 899-907, 1991.

18. Taguchi T, Wakui A, Hasegawa K, et al: Phase I clinical study of CPT-11. Research group of CPT-11. Jpn J Cancer Chemother 17: 115-120, 1990.

19. Slatter JG, Schaaf LJ, Sams JP, et al: Pharmacokinetics, metabolism, and excretion of irinotecan (CPT-11) following I.V. infusion of [(14)C]CPT-11 in cancer patients. Drug Metab Dispos 28: 423-433, 2000. 